\title{
The effects of kinesio taping of lower limbs on functional mobility, spasticity, and range of motion of children with spastic cerebral palsy
}

\author{
Mirjavad Tabatabaee ${ }^{1}$, Moslem Cheraghifard ${ }^{1}$ and Alireza Shamsoddini ${ }^{2^{*}}$ (D)
}

\begin{abstract}
Background: Cerebral palsy (CP) is a non-progressive neurological disease in a growing brain.

Objective: The aim of this study was to explore the effects of kinesio taping (KT) of the lower limbs on functional mobility, spasticity, and range of motion (ROM) of children with cerebral palsy.

Methods: Thirty children with spastic cerebral palsy aged 3 to 10 years old were selected from pediatric rehabilitation clinics. Subjects were divided randomly into intervention and control groups. Each group contains 15 subjects. Both groups received kinesio taping along with occupational therapy for 2 weeks. In the intervention group, kinesio taping was applied in a treatment mode with suitable tension; however, taping was applied in the control group in a sham mode. Timed get up and go (TUG), Modified Modified Ashworth (MMA), and goniometer tests were used prior to the intervention and 2 days and 2 weeks after the intervention.

Results: Short-term application of kinesio taping in the intervention group did not lead to significant changes in ROM $(P=0.582)$, muscle tone $(P=0.317)$, and functional mobility $(P=0.320)$. However, long-term application of kinesio taping improved the range of motion, muscle tone, and functional mobility $(P<0.05)$. No significant change has been observed in the control group in different intervals.

Conclusion: The findings indicated that kinesio taping can increase ROM of the knee and reduce spasticity. It also can improve the functional mobility. Therefore, it seems that kinesio taping is efficient for rehabilitation of spastic $\mathrm{CP}$ as a reliable treatment method.
\end{abstract}

Trial registration: IRCT, IRCT2017082135822N1, Registered 19 September 2017, https://fa.irct.ir/IRCT2017082135822N1.

Keywords: Cerebral palsy, Spasticity, Range of motion, Functional mobility, Kinesio taping

\section{Introduction}

Cerebral palsy $(\mathrm{CP})$ is a non-progressive neurological disorder that affects a child's motor skills, movements, and muscle tone. Cerebral palsy is caused by brain damage and develops while the baby is still in utero, during labor and delivery, or shortly after birth [1]. Cerebral palsy can result from different kind of disorders, such as brain growth, metabolic, genetic, and ischemic causes. Cerebral palsy is divided into hemiplegia, diplegia, and

\footnotetext{
* Correspondence: alirezaot@bmsu.ac.ir

2Exercise Physiology Research Center, Lifestyle Institute, Baqiyatallah

University of Medical Sciences, Tehran, Iran

Full list of author information is available at the end of the article
}

quadriplegia types based on the type of the involved part and is divided into spastic, dyskinesia, and hypotonic types based on the type of motion disorder. In a study in Iran, diplegia is proved to be the most prevalent type of cerebral palsy. These disorders are reported to be the most prevalent motion disorders in children with an outbreak of two per thousand [2]. Prevalent motion disorders in cerebral palsy children include abnormal muscle tone, survival of primary reflection patterns, or their intensification in voluntary movements, body awareness, and body schema disorders caused by incomplete sensory information [2]. The main challenge for spastic $\mathrm{CP}$ is gross motor dysfunction. The motor 
problems of spastic CP arise fundamentally from central nervous system dysfunction, which interferes in the development of normal alignment postural control against gravity and impedes normal motor development $[1,3]$.

The main goal of occupational therapy in the rehabilitation of cerebral palsy is to improve motor function and increase the ability to control movement [4]. The aim of functional mobility from one place to another is to fulfill an activity or duty which is exposed to problems in cerebral palsy children [4]. Functional mobility disorder in cerebral palsy children results from spasticity, paresis, sensory integrity damage, lack of selective control motor, and lack of coordination between agonist and antagonist muscles. Spasticity is considered to be the most important disorder in cerebral palsy which leads to muscle shortness, reduced range of joint motion, and functional mobility disorder after a while $[4,5]$. Kinesio taping can influence the skin, circulatory system, and motor control and leads to enhanced proprioception sense, reduced muscle spasticity, and strengthened muscles [3]. One of the solutions to treat spasticity is increased local blood flow. So, it can be inferred that kinesio taping reduces spasticity by improved blood flow [5-8]. On the other hand, kinesio taping reduces excessive tension and contraction to improve muscle tone and reduce spasticity [9]. It also improves motion and comfort; therefore, it can be used for cerebral palsy children efficiently [10]. Spasticity reduces functional mobility of cerebral palsy children after reducing their range of motion. Given the importance of motion and functional mobility in daily life, design and execution of accurate scientific interventions is necessary for health care systems economically and ethically. Research on this field and accurate analysis of functional mobility, spasticity, and range of motion (ROM) are scarce; therefore, the present study aims to explore the effects of kinesio taping of lower limbs on functional mobility, spasticity, and ROM of children with spastic cerebral palsy.

\section{Subjects and methods}

Thirty children with diplegia and quadriplegia spastic cerebral palsy, aged 4 to 10 , participated in this study. The samples were selected from the patients referring to pediatric rehabilitation clinics of Tehran. Subjects were divided randomly into intervention and control groups, each containing 15 subjects. This clinical trial was registered at the Iranian Registry of Clinical Trials of the Ministry of Health and Medical Education, Registered 19 September 2017, https://fa.irct.ir/ IRCT2017082135822N1. At first, subjects' parents or their relatives completed the biographical forms. Then, the specified tests and evaluations were carried out by the researcher in the clinic. Inclusion criteria included the following: (1) diagnosis of spastic cerebral palsy by the neurologist, (2) walking ability, (3) ability of understanding and executing the orders, (4) age ranging from 4 to 10 years old, (5) no other rehabilitation intervention, (6) no orthopedic abnormality. Exclusion criteria included the following: (1) a new intervention in addition to previous ones for these children, (2) absence in two taping change sessions, (3) skin sensitivity after taping (if the skin of each subjects was allergic to the kinesio taping, he/she was excluded), (4) children with spastic cerebral palsy with fixed knee flexion; (5) children who have had seizures for the past six months, (6) BTX-A injection for the past 6 months, (7) spastic CP patients have some dystonic elements. Both groups underwent physiotherapy and occupational therapy along with kinesio taping. Taping is applied on the ankles and tibialis muscles for improvement of dorsi flexion, on gastrocnemius muscle for inhibition of plantar flexion of ankles, on rectus femoris for improvement of knee extension, and on hamstring muscles for inhibition of knee flexion. Therefore, tension was applied to all the muscles. However, in inhibitory actions, it was applied from the end toward the beginning of the muscle. Moreover, the tension of tape in the muscular area was 30\% of the primary length and in the joint area was $75 \%$ of the primary length (Fig. 1).

Kinesio taping intervention was applied in the control group as in the intervention group, with the difference that no tension was applied to the kinesio tape. Therefore, in the control group, kinesio taping was performed without any tension (sham taping). The purpose of KT application using this procedure was to improve the function of weak muscles and decrease tonicity of the spastic muscles. The position of the child during taping was such that the muscle was tensile. Taping was done for 14 days, $24 \mathrm{~h}$ a day, on both feet, and it was changed every 4 days. The tests and evaluations were carried out and compared at intervals of before, 2 days after, and 14 days after taping, namely at the end of interventions. In this study, ROM in dorsi flexion and knee extension were estimated and recorded via a triangle (set square) meter. Herrero and colleagues analyzed the reliability of the triangle meter in cerebral palsy children and concluded that pre- and posttest reliabilities were good; however, intertester reliability was weak [11].

Spasticity was evaluated and recorded by Modified Modified Ashworth Scale which has five items, from 0 (normal muscle tone) to 4 (totally rigid organ). Reliability of this test was evaluated by Ghotbi for hip adductors, knee extensors, and ankle plantar flexion $[12,13]$. Functional mobility of children was evaluated via timed get up and go (TUG) test and its results were recorded. In this test, the duration of standing up from the chair, walking up to $3 \mathrm{~m}$, returning, and sitting on the chair was measured. Also, an elastic goniometer was used for the measurement of ROM. An informed consent was obtained from all parents of children including 

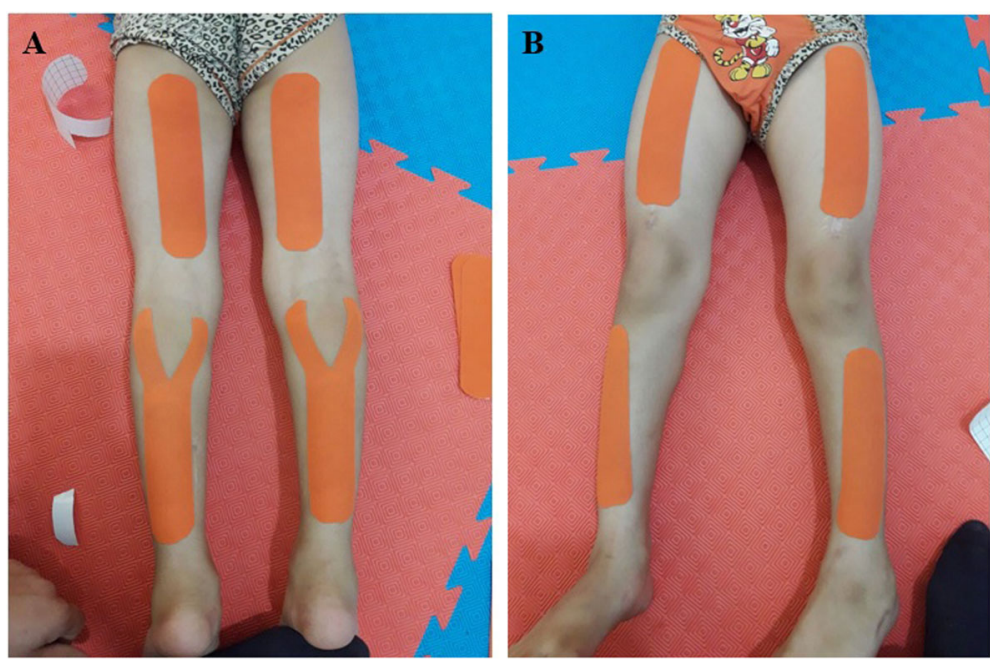

Fig. 1 Kinesio taping method used in this study. a Posterior view of kinesio taping and $\mathbf{b}$ anterior view of kinesio taping

agreement of the children to participate as volunteers. The study protocol was approved by the Human Research Ethics Committees of the Baqiyatallah University of Medical Sciences (IR.BMSU.REC.1396.526).

\section{Statistical analysis}

The Shapiro-Wilk test was used for normal distribution of studied variables and data. For comparison of distance variables in each group before and after interventions, paired $T$-test and, for comparison of intervention data in the two different groups, independent $T$-test were used. Moreover, time (before, after 2 days, and at the end of intervention), group (control and intervention), and their interactive main effects were measured via two-way repeated measures ANOVA. Wilcoxon signed-rank statistical test was applied for the comparison of ranked variables (muscle tone). Analyses were completed using the Statistical Package for Social Sciences (SPSS Inc. Chicago, IL, USA) version 20.0 software, $2011(P<0.05)$.

\section{Results}

Thirty children with cerebral palsy participated in this study. Fifteen patients with average age of 7 were put in the intervention group, and 15 patients with average age of 6.93 were in the control group. The participants in the intervention group were 10 males and 5 females and in the control group were 8 males and 7 females. 53.3\% of the participants in both groups had diplegia, and $47.7 \%$ had quadriplegia.

Mean and standard deviation of the evaluated variables are indicated in Table 1. In primary evaluation of ankle ROM, scores of TUG test and ankle and knee spasticity indicated no significant difference between the two groups. However, knee ROM indicated a significant difference between the two groups $(P<0.001)$. Table 2 represents ANOVA test results. Time (before, after 2 days, and after 2 weeks) and interactive main effects (group $\times$ time) for all the three variables were significant; however, group effects (intervention and control) were only significant for knee ROM. Triple evaluations of changes in each group indicated that there was a significant difference between the first and second evaluations of ankle ROM in the intervention group $(P=0.58)$. However, no significant difference was observed in comparing the before and after intervention results $(P<0.001)$. A significant difference was observed between the first and second $(P=0.656)$ evaluation with the first and third $(P=0.77)$ evaluation results in the control group.

No significant difference was observed between the first and second evaluation results of knee ROM in the intervention group $(P=0.43)$; however, there was a significant difference between the first and third evaluation results $(P<0.001)$. Moreover, no significant difference was observed between the first and second $(P=0.22)$ evaluation with first and third $(P=0.13)$ evaluation results.

The evaluation results of the TUG test indicated no significant difference between the first and second evaluation results of the intervention group $(P=0.32)$; however, a significant difference was observed between the first and third evaluation results $(P<0.001)$. No significant difference was observed between the first and second $(P=0.33)$ evaluation with first and third $(P=0.43)$ evaluation results.

Short-term application of kinesio taping in the ankle muscle tone indicated no significant change in the intervention $(P=0.31)$ and control $(P=0.21)$ groups. However, the primary and final evaluation of the ankle muscle tone $(P=0.014)$ indicated a significant change in the intervention group, but no significant change was recorded in the control group $(P=0.15)$. 
Table 1 Mean and standard deviation of ROM and functional mobility in two groups

\begin{tabular}{|c|c|c|c|c|}
\hline \multirow[t]{2}{*}{ Variables } & \multirow[t]{2}{*}{ Group } & \multicolumn{3}{|l|}{ Time } \\
\hline & & Before & After 2 days & After 2 weeks \\
\hline \multirow[t]{2}{*}{ ROM of knee (degree) } & Control & $118 / 86 \pm 5 / 66$ & $119 \pm 5 / 81$ & $121 / 86 \pm 6 / 35$ \\
\hline & Intervention & $125 / 33 \pm 3 / 90$ & $125 / 33 \pm 3 / 82$ & $125 / 73 \pm 4 / 09$ \\
\hline$P$ value & & $<0.001$ & $<0.001$ & 0.068 \\
\hline \multirow[t]{2}{*}{ ROM of ankle (degree) } & Control & $-10 / 40 \pm 1 / 80$ & $-10 / 33 \pm 1 / 95$ & $-7 / 93 \pm 3 / 01$ \\
\hline & Intervention & $-8 / 80 \pm 3 / 54$ & $-8 / 80 \pm 3 / 54$ & $-8 / 73 \pm 3 / 67$ \\
\hline$P$ value & & 0.131 & 0.154 & 0.520 \\
\hline \multirow[t]{2}{*}{ Functional mobility (second) } & Control & $14 / 46 \pm 3 / 54$ & $14 / 46 \pm 3 / 39$ & $12 / 46 \pm 2 / 97$ \\
\hline & Intervention & $15 / 46 \pm 3 / 18$ & $15 / 33 \pm 2 / 99$ & $15 / 60 \pm 3 / 13$ \\
\hline$P$ value & & 0.423 & .0465 & 0.009 \\
\hline
\end{tabular}

ROM range of motion

Short-term application of kinesio taping in knee muscle tone indicated no significant change in the intervention $(P=0.31)$ and control $(P=0.25)$ groups. However, the primary and final evaluations of knee muscle tone $(P=0.014)$ indicated a significant change in the intervention group, but no significant change was observed in the control group $(P=0.31)$.

\section{Discussion}

The aim of this study was to analyze the effects of kinesio taping on ROM, spasticity, and functional mobility of diplegia and quadriplegia spastic cerebral palsy children. The results indicated no significant difference after 2 days of intervention; however, they indicated a significant difference after 2 weeks of intervention. Two weeks after the intervention, a significant improvement in knee and knee ranges of motion and a reduction in spasticity have been observed.

Spasticity is considered the most important functional disorder of cerebral palsy, which is followed by muscle shortness and reduced range of joint mobility along with functional mobility disorders [14-16]. Interventions which are done with the aim of reducing spasticity can

Table 2 Time and interactive main effects for all the three variables

\begin{tabular}{llll}
\hline Variables & Effects & $F$ & $P$ value \\
\hline ROM of knee (degree) & Time & $34 / 18$ & $<0.001$ \\
& Group & $9 / 27$ & 0.005 \\
& Time $\times$ group & $19 / 76$ & 0.001 \\
ROM of ankle (degree) & Time & $24 / 28$ & $<0.001$ \\
& Group & $0 / 521$ & 0.476 \\
& Time $\times$ group & $21 / 76$ & $<0.001$ \\
Functional mobility (Second) & Time & $28 / 23$ & $<0.001$ \\
& Group & 205 & 0.162 \\
& Time $\times$ group & $42 / 11$ & $<0.001$ \\
\hline
\end{tabular}

ROM range of motion lead to improved ROM and, consequently, improved functional mobility [17]. Spasticity of the hamstring and gastrocnemius muscles causes disability in ankle dorsiflexion and knee extension [18]. It seems that kinesio taping can inhibit spastic muscles by applying a retentive tension on these muscles and changing the mechanic properties of the muscles and other soft tissues [19, 20].

Kinesio taping can make changes by creating nervous feedback in the patients and facilitate motion by mechanic induction of soft tissues. Consequently, it can improve ROM [21, 22]. Different kinds of hypotheses are presented to improve the range of motion, such as pain reduction, and improve muscle alignment [23]. One of the hypotheses argues that by applying the tape, the skin will be stretched due to the flexibility of the tape, and the distance of the skin with muscle will be increased which increases local blood and lymph flow and, consequently, improves the functional mobility and ROM [21].

Positive effects of kinesio taping for improving ROM in patients with different disorders are reported in some of the studies. In the present study, kinesio taping along with physiotherapy and occupational treatment is considered an effective method for ankle and knee ROM improvement in cerebral palsy children. In a study, Choi conducted a similar research on the effects of ankle kinesio taping before and after proprioceptive neuromuscular facilitation (PNF) exercises in patients with stroke. Their results indicated a significant increase in dorsi flexion ROM and Berg balance scale, and a significant reduction in TUG duration [24].

Yoshida and colleagues analyzed the effects of kinesio tape on lower limbs' range of motion. Their study sample consisted of 30 healthy people without any lower limb profile or backache. They concluded that flexion difference was very significant; therefore, flexion ROM increases after applying kinesio tape. These results are consistent with the results of the present study [25]. 
Demirel recruited 15 cerebral palsy children, aged 6 to 18 years old, to analyze the effects of kinesio taping on wrist active range of motion. Kinesio taping was applied with functional technique on a child's wrist. Before and after intervention evaluations were measured by a triangle meter. The researchers concluded that extension active range of motion, radial deviation, and ulnar deviation were improved after interventions [26]. Greve and colleagues conducted a case study to analyze the effects of kinesio taping on gastrocnemius muscle which leads to spasticity reduction in diplegia spasticity cerebral palsy children. They observed a significant difference in the Modified Modified Ashworth (MMA) Scale. Their results are consistent with the results of the present study [27]. Karadag and colleagues used a combination of kinesio taping and Botox gastrocnemius for patients with stroke and observed significant changes, such as an improved MMA scale, improved walking speed, and increased step length [8]. Functional mobility requires muscle strength, motor coordination, and ability of changing status while maintaining stability. In the present study, functional mobility was evaluated by TUG which indicated significant changes in a shorter period after interventions. In a similar study, Kim and colleagues used kinesio taping for 26 patients with stroke along with their regular physiotherapy schedules. After 8 weeks, they reported that kinesio taping could significantly reduce TUG duration. However, in this study, kinesio taping was applied three times a week for 30 min, while in our study, it was applied for $24 \mathrm{~h}$ on patients' feet [28].

\section{Conclusions}

Based on the results of this study (long-term affectability) and other researches, kinesio taping is effective for spastic cerebral palsy children and can improve their ROM and functional mobility, and reduce their spasticity. Therefore, kinesio taping could be used as an add-on method for rehabilitation of children with spastic cerebral palsy.

\section{Abbreviations}

CP: Cerebral palsy; KT: Kinesio taping; ROM: Range of motion; TUG: Timed get up and go; MMA: Modified Modified Ashworth

\section{Acknowledgements}

The authors acknowledge the subjects for their participation and cooperation in this study.

\section{Authors' contributions}

AS and MT contributed to the study concept and design. MT and MC contributed to the acquisition of data. MT and MC contributed to the analysis and interpretation of data. AS and MT contributed to the drafting of the manuscript. AS contributed to the editing of the manuscript. All authors read and approved the final manuscript.

\section{Funding}

This research received no specific grant from any funding agency in the public or commercial sector.

\section{Availability of data and materials}

The datasets used and/or analyzed during the current study are available from the corresponding author on reasonable request.

\section{Ethics approval and consent to participate}

At the beginning of this study, an informed consent was obtained from all parents of children, including an agreement of the children to participate as volunteers. This study is confirmed by the ethical committee of Baqiyatallah University of Medical Sciences in November 2017 with Ethics code: IR.BMSU.REC.1396.526.

\section{Consent for publication}

Not applicable

\section{Competing interests}

The authors declare that they have no competing interests.

\section{Author details}

'Department of Occupational Therapy, School of Rehabilitation, Iran University of Medical Sciences, Tehran, Iran. ${ }^{2}$ Exercise Physiology Research Center, Lifestyle Institute, Baqiyatallah University of Medical Sciences, Tehran, Iran.

Received: 26 February 2019 Accepted: 6 September 2019

Published online: 29 October 2019

\section{References}

1. Rosenbaum P, Paneth N, Leviton A, Goldstein M, Bax M, Damiano D, et al. A report: the definition and classification of cerebral palsy April 2006. Dev Med Child Neurol. 2007;109(suppl 109):8-14.

2. Joghataei MT, Mohammad K, Rahgozar M, Siadati S. Prevalence of some paralysis and limb amputation disabilities in Iran national epidemiological survey. J Archives of Rehabilitation. 2002;3(1):7-16.

3. Mackin EJ, Hunter JM. Rehabilitation of the hand and upper extremity: Mosby; 2002. p. 232-56.

4. Podsiadlo D, Richardson S. The timed "Up \& Go": a test of basic functional mobility for frail elderly persons. J Am Geriatr Soc. 1991;39(2):142-8.

5. Bar-On L, Molenaers G, Aertbeliën E, Van Campenhout A, Feys H, Nuttin B, et al. Spasticity and its contribution to hypertonia in cerebral palsy. 2015; 2015

6. Dingwell JB, Marin LC. Kinematic variability and local dynamic stability of upper body motions when walking at different speeds. J Biomech. 2006; 39(3):444-52.

7. Tagliabue M, Ferrigno G, Horak F. Effects of Parkinson's disease on proprioceptive control of posture and reaching while standing Neuroscience. 2009:158(4):1206-14.

8. Karadag-Saygi E, Cubukcu-Aydoseli K, Kablan N, Ofluoglu D. The role of kinesio taping combined with botulinum toxin to reduce plantar flexors spasticity after stroke. Top Stroke Rehabil. 2010;17(4):318-22.

9. Shamsoddini A, Hollisaz MT, Hafezi R. Initial effect of taping technique on wrist extension and grip strength and pain of individuals with lateral epicondylitis. Iran Rehab J. 2010;8(11):24-8.

10. Gordon AM, Bleyenheuft Y, Steenbergen B. Pathophysiology of impaired hand function in children with unilateral cerebral palsy. Dev Med Child Neurol. 2013;55(s4):32-7.

11. Herrero PCP, Garcia E, Gomez-Trullen EM, Olivan-Blazquez B. Reliability of goniometric measurements in children with cerebral palsy: a comparative analysis of universal goniometer and electronic inclinometer. A pilot study. BMC musculoskeletal disord. 2011:12:115.

12. Ghotbi N, Ansari NN, Naghdi S, Hasson S, Jamshidpour B, Amiri S. Inter-rater reliability of the Modified Modified Ashworth Scale in assessing lower limb muscle spasticity. 2009; 23(10):815-819.

13. Cattaneo D, Jonsdottir J, Repetti S. Reliability of four scales on balance disorders in persons with multiple sclerosis. Disabil Rehabil. 2007;29(24): $1920-5$.

14. Tilton AH. Management of spasticity in children with cerebral palsy. Seminars in Pediatric neurology. 2004;11(1):58-65.

15. Kaya Kara O, Atasavun Uysal S, Turker D, Karayazgan S, Gunel MK, Baltaci G. The effects of kinesio taping on body functions and activity in unilateral spastic cerebral palsy: a single-blind randomized controlled trial. Dev Med Child Neurol. 2015;57(1):81-8. 
16. Stempien L, Tsai T. Intrathecal baclofen pump use for spasticity: a clinical survey. Am J Phys Med Rehabil. 2000;79(6):536-41.

17. Park E-YJJopts. Effect of physical therapy frequency on gross motor function in children with cerebral palsy. 2016;28(6):1888-1891.

18. Ryerson SD. The foot in hemiplegia. In: Physical Therapy of the Foot and Ankle. New York, NY: Churchill-Livingstone; 1988. p. 109-31.

19. Hufschmidt A, Mauritz K. Chronic transformation of muscle in spasticity: a peripheral contribution to increased tone. J Neurol Neurosurg Psychiatry. 1985:48(7):676-85.

20. Labbf S, Shamsoddini A, Hollisaz MT, Sobhani V, Shakibaee A. Effects of neurodevelopmental therapy on gross motor function in children with cerebral palsy. Iran J Child Neurol. 2015;9(2):36-41.

21. Kase K. Clinical therapeutic applications of the kinesio taping method. Albuquerque. 2003:25-60.

22. Lee $Y$-Y, Chang $\mathrm{H}-\mathrm{Y}$, Chang $\mathrm{Y}-\mathrm{C}$, Chen J-M. The effect of applied direction of kinesio taping in ankle muscle strength and flexibility. In: ISBS-Conference Proceedings Archive; 2012.

23. Amirsalari S, Dalvand H, Dehghan L, Feizy A, Hosseini SA, Shamsoddini A. The efficacy of botulinum toxin type A injection in the hamstring and calf muscles with and without serial foot casting in gait improvement in children with cerebral palsy. Tehran Univ Med J. 2011;69(8):509-17.

24. Choi Y-K, Nam C-W, Lee J-H, Park Y-H. The effects of taping prior to PNF treatment on lower extremity proprioception of hemiplegic patients. J Phys Ther Sci. 2013;25(9):1119-22.

25. Yoshida A, Kahanov $L$. The effect of kinesio taping on lower trunk range of motions. Research in sports medicine. Res Sports Med. 2007:15(2):103-12.

26. Demirel A, Bayrakci VT. The effect of kinesio tape on active wrist range of motion in children with cerebral palsy: a pilt study. J Orthop. 2014;6(2):69-74.

27. Greve P, Perez V, Yoshizumi L, Morini N, Faria T, Berzin F. Effect of the bandage kinesio taping ${ }^{\odot}$ in spasticity in cerebral palsy of diparetic-case report. Proceedings of the 14th World Physical Therapy. 2003:7-12.

28. Kim YR, Kim Jl, Kim YY, Kang KY, Kim BK, Park JH, et al. Effects of ankle joint taping on postural balance control in stroke patients. J Int Acad Phys Ther Res. 2012;3(2):446-52.

\section{Publisher's Note}

Springer Nature remains neutral with regard to jurisdictional claims in published maps and institutional affiliations.

\section{Submit your manuscript to a SpringerOpen ${ }^{\circ}$ journal and benefit from:}

- Convenient online submission

- Rigorous peer review

- Open access: articles freely available online

- High visibility within the field

- Retaining the copyright to your article

Submit your next manuscript at $\boldsymbol{\nabla}$ springeropen.com 